\title{
Supersymmetric Reflection Matrices *
}

\author{
M. Moriconi ${ }^{\dagger}$ \\ High Energy Section, ICTP \\ Strada Costiera 11, 34100 Trieste, Italy \\ K. Schoutens $\ddagger$ \\ Institute for Theoretical Physics, University of Amsterdam \\ Valckenierstraat 65, 1018 XE Amsterdam, The Netherlands
}

\begin{abstract}
We briefly review the general structure of integrable particle theories in $1+1$ dimensions having $N=1$ supersymmetry. Examples are specific perturbed superconformal field theories (of Yang-Lee type) and the $N=1$ supersymmetric sine-Gordon theory. We comment on the modifications that are required when the $N=1$ supersymmetry algebra contains non-trivial topological charges.
\end{abstract}

$\mathrm{IC} / 97 / 34$

ITFA-97-13

${ }^{*}$ Talk given at the International Seminar on Supersymmetry and Quantum Field Theory, dedicated to the memory of D.V. Volkov, Kharkov (Ukraine), January 5-7, 1997

${ }^{\dagger}$ e.mail: moriconi@ictp.trieste.it

‡e.mail: kjs@phys.uva.nl 


\section{INTRODUCTION}

Quantum field theory (QFT) provides a powerful and unifying language to understand a variety of physical phenomena. In general we may define a QFT by choosing a set of fields that transform according to some irreducible representation of the Poincaré group, together with a prescription (for example, coming from a lagrangian) that gives us the dynamics of these fields. In the context of Particle Physics, it is strongly believed that the Poincaré group is a true symmetry of the world and therefore we always take it for granted.

In general, a QFT becomes more tractable if it possesses additional symmetries beyond Poincaré invariance. Via Ward identities, extra symmetries provide strong constraints on correlation functions and so make possible a more thorough analytical treatment. To introduce new symmetries in a QFT is rather delicate: we do not want to oversimplify the specific models we are looking at but we would like to have enough symmetry to improve the physical properties and to gain the upper hand in controlling the theory. Among the possible symmetries we may consider, supersymmetry stands out as a very special one. Supersymmetry unifies the apparently incompatible concepts of bosons and fermions, and often improves the physical properties of specific field theories. At the same time, supersymmetric theories are usually easier to analyze. This last remark applies to supersymmetric Yang-Mills theory (in 4 dimensions), to string models (in 10 dimensions) and to models of QFT in 2 dimensions.

Focussing on QFT's in $1+1$ dimensions, we may further specialize to models that are integrable. By this we mean that we assume the existence of 'enough' (meaning an infinite number of) charges in involution.] For a QFT describing (massive) particles, integrability implies the factorizability of the scattering matrix: the S-matrix for $n$ particle scattering factorizes into two-body S-matrices, there is no particle production and the individual momenta of the particles are conserved. By assuming both supersymmetry and integrability, we thus arrive at very simple supersymmetric particle theories which can be analyzed in closed form, and which may serve as prototypes for supersymmetric particle theories in higher dimensions.

In addition to the motivation we have given so far, there are more direct reasons for considering QFT's in two dimensions. For one thing, such theories directly apply to the analysis of either classical systems of statistical mechanics in 2 dimensions, or quantum mechanical systems in $1+1$ dimensions (that is, on a line). In addition, there are applications to problems in $3+1$ dimensions, where the essential physics takes place in the radial direction. In the latter type of applications, the models live on a half-line and the behavior at the boundary ${ }^{2}$ is important. Examples are the Callan-Rubakov effect (the catalysis of baryon decay in the field of a magnetic monopole), the Hawking effect (quantum black-hole evaporation), the Kondo problem (magnetic impurities coupling to conduction electrons) or edge current tunneling in the quantum Hall effect.

In view of these applications, it is an interesting problem to consider integrable supersymmetric particle theories in $1+1$ dimensions in the presence of a boundary. In a recent paper [i] we obtained a general form for boundary reflection matrices in $N=1$ supersymmetric theories, and we worked out a number of examples.

In this note we outline the general structure of integrable supersymmetric QFT's in $1+1$ dimensions, paying particular attention to their boundary scattering. We shall briefly introduce specific examples (which are perturbations of supersymmetric Yang-Lee-type conformal field theories and the breathers in the supersymmetric sine-Gordon theory). We shall also comment on the extension of these results to the case of $N=1$ supersymmetry with non-zero topological charge.

\section{S-MATRICES AND REFLECTION MATRICES: GENERAL}

Given a bulk integrable field theory, one may start the analysis by determining the two-body scattering matrix. This is a key ingredient for the understanding of the physics of the model and the first step towards computing correlation functions using the form-factor approach. From now on the term "S-matrix" will be used for the two-body S-matrix unless stated otherwise explicitly. We will use the rapidity variable $\theta$, which parametrizes the on-shell momenta of the particles by $p_{0}=m \cosh (\theta)$ and $p_{1}=m \sinh (\theta)$. The S-matrix between particles 1 and 2 can be written as $\left.S_{a_{1} a_{2}}^{b_{1} b_{2}}\left(\theta_{12}\right)\right)$, where $\theta_{12}=\theta_{1}-\theta_{2}$ is the difference of the rapidities of the incoming particles.

Let us now briefly outline the general strategy for obtaining the S-matrix for an integrable model with some nontrivial symmetries. One starts by writing down the most general S-matrix compatible with the unbroken symmetries

\footnotetext{
${ }^{1}$ To say that charges are in involution means (in classical mechanics) that the Poisson brackets of any two of them vanish or (in QFT) that they all mutually commute.

${ }^{2}$ the origin of space in the original three-dimensional formulation
} 
and then requires integrability. This is done by imposing the famous Yang-Baxter equation (YBE). The YBE is shown in figure 1 .
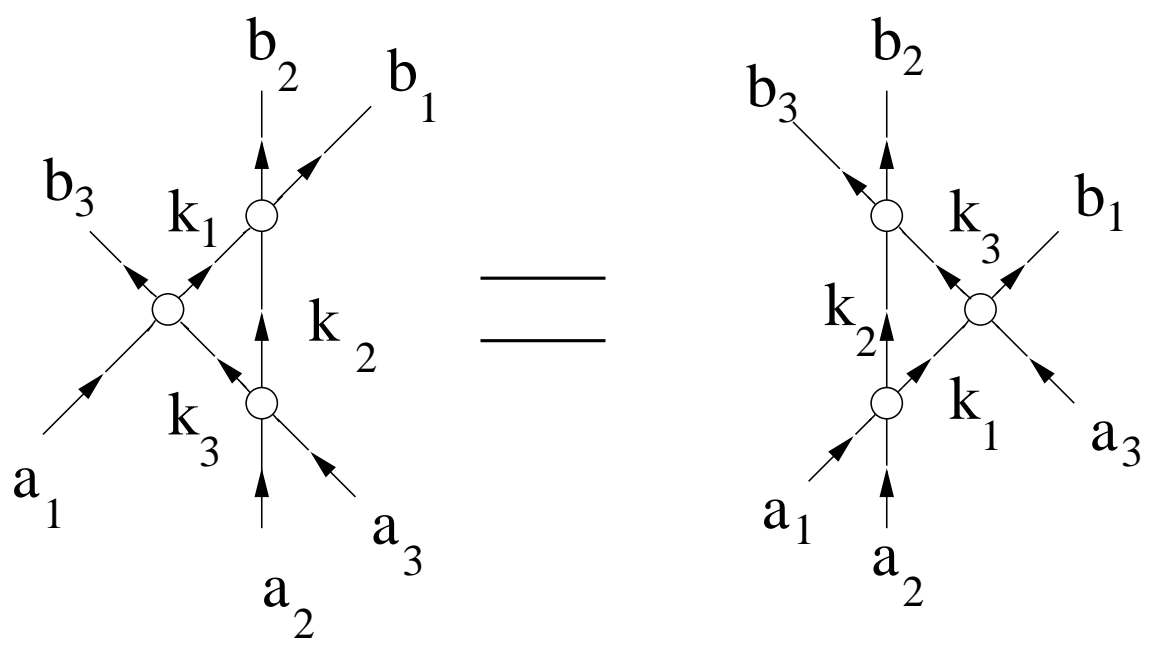

Fig. 1 Pictorial representation of the YBE

In a formula the YBE reads

$$
\begin{aligned}
& \sum_{k} S_{a_{1} a_{2}}^{k_{1} k_{2}}\left(\theta_{12}\right) S_{k_{1} a_{3}}^{b_{1} k_{3}}\left(\theta_{13}\right) S_{k_{2} k_{3}}^{b_{2} b_{3}}\left(\theta_{23}\right)= \\
= & \sum_{k} S_{a_{2} a_{3}}^{k_{2} k_{3}}\left(\theta_{23}\right) S_{a_{1} k_{3}}^{k_{1} b_{3}}\left(\theta_{13}\right) S_{k_{1} k_{2}}^{b_{1} b_{2}}\left(\theta_{12}\right) .
\end{aligned}
$$

Once the YBE is solved we impose the usual constraints from general S-matrix theory, that is, analyticity, crossingsymmetry and unitarity. After we managed to do all that (it can be done in many cases!) we have to impose the so-called bootstrap principle: bound states are to be treated on the same footing as asymptotic states. This, together with integrability as encoded in the YBE, provides a very restrictive set of equations that greatly constrain the initial S-matrix. Once we reach a self-consistent spectrum we will have found the minimal S-matrix for our model. Of course this can not be the whole story, since different models with the same symmetry and same spectrum may correspond to quite different lagrangians, say. This ambiguity is indeed present and it is called CDD ambiguity, after the work of Castillejo, Dalitz and Dyson [2].

One of the ways to test a conjectured S-matrix is through the thermodynamic Bethe Ansatz (TBA). This is a general procedure where we start with the S-matrix as input and compute some ultraviolet physical properties such as the ground state energy (central charge) and scaling dimensions of the underlying QFT. Comparing these with ultraviolet data obtained from a lagrangian or from conformal field theory, we have a non-trivial check on the conjectured S-matrix.

Next we go from the bulk theory to a theory defined on half-line. We will have to specify the boundary action or simply assume that the boundary action is such as to preserve integrability and the extra symmetries of the theory. The theory is then described in the bulk by the same S-matrix as before but now we have to find reflection matrices $R_{a}^{b}(\theta)$, which tell us how particles scatter off the boundary. We will assume that the boundary does not change the particle species, so that $R_{a}^{b}(\theta)=\delta_{a}^{b} R_{a}(\theta)$. Integrability is imposed now via the boundary Yang-Baxter equation (BYBE), which can be represented in this case as 

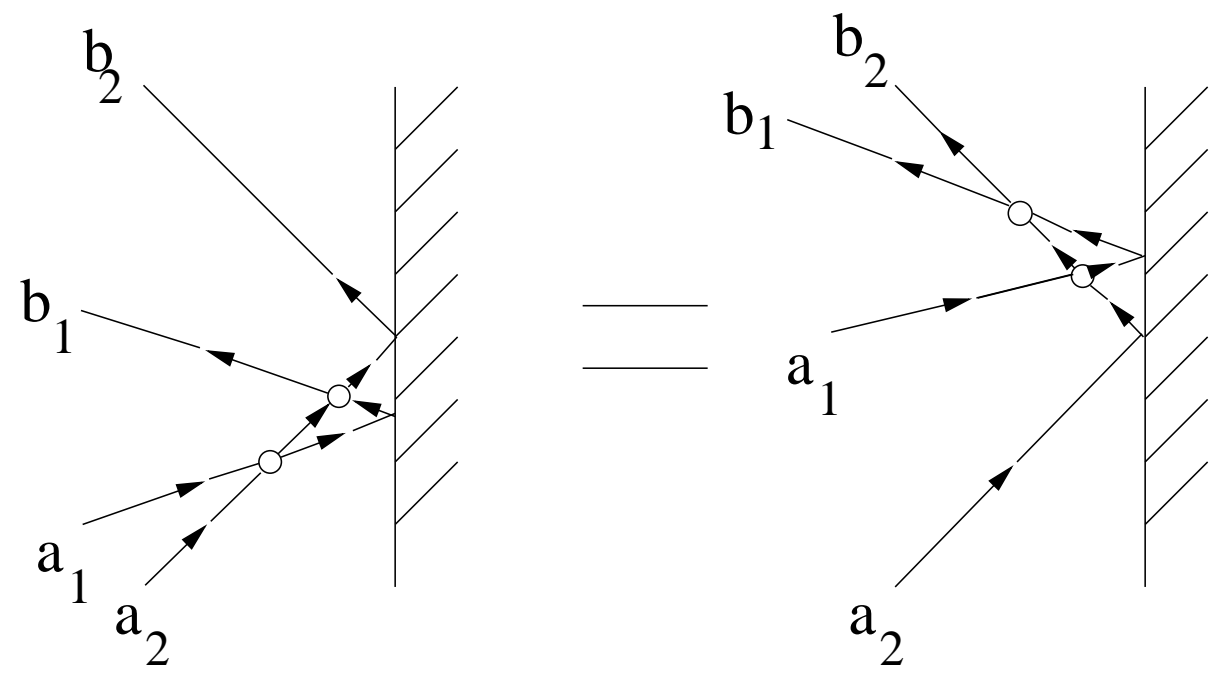

Fig. 2 Pictorial representation of the BYBE

In a formula|',

$$
\begin{aligned}
& R_{a_{2}}\left(\theta_{2}\right) S_{a_{1} a_{2}}^{c_{1} d_{2}}\left(\theta_{1}+\theta_{2}\right) R_{c_{1}}\left(\theta_{1}\right) S_{d_{2} c_{1}}^{b_{2} b_{1}}\left(\theta_{1}-\theta_{2}\right)= \\
& \quad S_{a_{1} a_{2}}^{c_{1} c_{2}}\left(\theta_{1}-\theta_{2}\right) R_{c_{1}}\left(\theta_{1}\right) S_{c_{2} c_{1}}^{b_{2} b_{1}}\left(\theta_{1}+\theta_{2}\right) R_{b_{2}}\left(\theta_{2}\right) .
\end{aligned}
$$

The general idea is clear now. Once we found an exact (bulk) S-matrix we can study boundary versions of the same theory by solving the BYBE and the analogous requirements on the reflection matrix (analyticity, unitarity, boundary crossing-unitarity). Note that the introduction of a boundary necessarily changes the structure of conserved charges. Some of the charges that were conserved in the bulk are not conserved anymore, e.g., linear momentum.

\section{III. $N=1$ SUPERSYMMETRY WITHOUT TOPOLOGICAL CHARGES}

Supersymmetry may sound as an odd concept in $1+1$ dimensions, since the very definition of bosons and fermions is based on the behavior of the matter field under the rotation group. Since we do not have a rotation group in $1+1$ dimensions one may feel uneasy with such concepts. One may realize, however, that the Lorentz group (which is non-trivial in 1+1 dimension) suffices to define the notions of bosonic fields (of integer Lorentz spin) and fermionic fields (of half-integer Lorentz spin). Supersymmetry comes with a parity operator $Q_{L}$, which has eigenvalue +1 on bosonic states and -1 on fermionic states. The $N=1$ supersymmetry algebra takes the form

$$
\begin{gathered}
\left\{Q_{L}, Q_{ \pm}\right\}=0 \\
Q_{+}{ }^{2}=p_{0}+p_{1}, \quad Q_{-}{ }^{2}=p_{0}-p_{1} \\
\left\{Q_{+}, Q_{-}\right\}=0 .
\end{gathered}
$$

The anticommutator $\left\{Q_{+}, Q_{-}\right\}$could have been non-zero, and equal to a real number $Z \leq 2$, which would correspond to a topological charge $Z$. We will initially consider the situation $Z=0$ and later show how it can be generalized to $Z \neq 0$. We will use the following realization

$$
Q_{+}(\theta)=\sqrt{m} e^{\frac{\theta}{2}}\left(\begin{array}{ll}
0 & 1 \\
1 & 0
\end{array}\right), \quad Q_{-}(\theta)=\sqrt{m} e^{-\frac{\theta}{2}}\left(\begin{array}{cc}
0 & -i \\
i & 0
\end{array}\right), \quad Q_{L}(\theta)=\left(\begin{array}{cc}
1 & 0 \\
0 & -1
\end{array}\right) .
$$

We can then define how these operators act on multi-particle states (all we need are two-particle states) and impose that the S-matrix commutes with the supersymmetry generators [3]. There is one point here that we should stress: to date all exact $N=1 \mathrm{~S}$-matrices are of a special form, given by

\footnotetext{
${ }^{3}$ no sum over $a_{1}, a_{2}, b_{1}, b_{2}$
} 


$$
S^{[i j]}(\theta)=S_{B}^{[i j]}(\theta) S_{B F}^{[i j]}(\theta)
$$

where $S_{B}^{[i j]}(\theta)$ is the S-matrix of the bosonic projection of the theory and $S_{B F}^{[i j]}(\theta)$ is a universal S-matrix that mixes bosons and fermions in such a way that the final non-diagonal S-matrix commutes with the supersymmetry charges. The bosonic factor $S_{B}^{[i j]}(\theta)$, describing the scattering of bosons $b_{i}$ and $b_{j}$, is assumed to be diagonal. All the physical (bound-state) poles of the total S-matrix are contained in this factor. The fermions are labeled by $f_{i}$ with $i=$ $1,2, \ldots, n$. The particles $b_{i}$ and $f_{i}$ have the same mass and form a supermultiplet under the $N=1$ supersymmetry. The general $S_{B F}^{[i j]}$ matrix was proposed by one of us in [3] and we refer to that paper for a more complete discussion.

In [3] it was found that integrability and supersymmetry alone fix the form of $S_{B F}^{[i j]}(\theta)$ up to one constant $\alpha$. To fix this constant we have to look at the bootstrap relations. It can be shown that if the particles $b_{i}$ and $b_{j}$ for a bound state $b_{k}$ then we have the following relation

$$
\alpha=-\frac{\left(2 m_{i}^{2} m_{j}^{2}+2 m_{i}^{2} m_{k}^{2}+2 m_{j}^{2} m_{k}^{2}-m_{i}^{4}-m_{j}^{4}-m_{k}^{4}\right)^{\frac{1}{2}}}{2 m_{i} m_{j} m_{k}} .
$$

Another consequence of supersymmetry is that once we have a three-point coupling between particles $b_{i}, b_{j}$ and $b_{k}$ we will also have three-point couplings for $\left(f_{i}, f_{j}, b_{k}\right),\left(f_{i}, b_{j}, f_{k}\right)$ and $\left(b_{i}, f_{j}, f_{k}\right)$. The following ratio is then obtained

$$
\frac{f_{f_{i} f_{j} b_{k}}}{f_{b_{i} b_{j} b_{k}}}=\left(\frac{m_{i}+m_{j}-m_{k}}{m_{i}+m_{j}+m_{k}}\right)^{\frac{1}{2}}
$$

From conditions such as (3.4) it is clear that bosonic theories that can be supersymmetrized in this simple manner have to be rather special. In all known examples, the masses $m_{i}$ of the particles $b_{i}$ come out as

$$
m_{i}=\frac{\sin (i \beta \pi)}{\sin (\beta \pi)}, \quad \beta=\frac{1}{2 n+1},
$$

for $i=1,2, \ldots, n, \alpha=-\sin (\beta \pi)$, and there is a specific bound state structure, related to $A_{2 n}^{(2)}$ group theory. In section $\mathrm{V}$ we shall present some explicit examples.

\section{SUPERSYMMETRIC REFLECTION MATRICES}

In this section we will explain how to obtain boundary reflection matrices for $N=1$ supersymmetric theories by circumventing some of the typical difficulties of boundary integrable models. The basic assumption is that the boundary action is such that integrability and supersymmetry are both preserved. On top of that we assume that the reflection matrix can be factorized in a similar fashion to the bulk S-matrix,

$$
R(\theta)=R_{B}(\theta) R_{B F}(\theta) .
$$

The $R_{B}(\theta)$ factor is the reflection matrix for the bosonic projection of the theory, and the $R_{B F}(\theta)$ is the "supersymmetric" piece. This factor has the following representation in a $|b\rangle,|f\rangle$ basis

$$
R_{B F}(\theta)=\left(\begin{array}{cc}
R_{b b}(\theta) & R_{b f}(\theta) \\
R_{f b}(\theta) & R_{f f}(\theta)
\end{array}\right)
$$

As we will see now, this will be enough to fix almost completely the reflection matrices, in a similar way to what happens in the bulk case.

If we assume that supersymmetry is preserved by the boundary action we have to impose the "commutation" relation between the reflection matrix and some linear combination of the two bulk supercharges

$$
\mathcal{Q}(\theta) R(\theta)=R(\theta) \mathcal{Q}(-\theta),
$$

where $\mathcal{Q}(\theta)=a Q_{+}(\theta)+b Q_{-}(\theta), a$ and $b$ some arbitrary real numbers. It is easy to see that the only solutions for (4.3) are $a= \pm b$ and

$$
R_{B F}^{ \pm}(\theta)=Z^{ \pm}(\theta)\left(\begin{array}{cc}
\cosh \left(\frac{\theta}{2} \pm i \frac{\pi}{4}\right) & e^{i \frac{\pi}{4}} Y(\theta) \\
e^{-i \frac{\pi}{4}} Y(\theta) & \cosh \left(\frac{\theta}{2} \mp i \frac{\pi}{4}\right)
\end{array}\right)
$$


By imposing BYBE we find that $Y(\theta)=0$. This means that the boundary can not change the fermion number of the incoming particle.

The mass (supermultiplet) dependence of $R_{B F}^{( \pm)}$is encoded in the prefactor $Z^{( \pm)}$. So in order to have a complete description of boundary scattering we have to fix these functions, by imposing unitarity and boundary crossingsymmetry. This was done in [1] and we refer to that paper for details. On the other hand, without any further work we can see immediately that the ratio $R_{b} / R_{f}$ is universal

$$
\frac{R_{b}^{ \pm}(\theta)}{R_{f}^{ \pm}(\theta)}=\frac{\cosh \left(\frac{\theta}{2} \pm i \frac{\pi}{4}\right)}{\cosh \left(\frac{\theta}{2} \mp i \frac{\pi}{4}\right)} .
$$

These results follows directly from supersymmetry, the factorization Ansatz (4.1) and the specific realization of the superalgebra that we are using.

\section{EXAMPLES: SUPERSYMMETRIC YANG-LEE MODELS AND BREATHERS IN THE SUPERSYMMETRIC SINE-GORDON MODEL}

In this section we give some examples of theories that realize the general structure presented in sections III and IV.

The first series of examples are the so-called supersymmetric generalized Yang-Lee models. They are obtained as integrable deformations of specific $N=1$ superconformal field theories of central charges $c=-3 n(4 n+3) /(2 n+2), n=$ $1,2, \ldots$, where the perturbing field is the bottom component of the Neveu-Schwarz field labeled as $\phi_{(1,3)}$. The spectrum of the massive deformation is as in (3.6). The first model of this series corresponds to the supersymmetrization of the Yang-Lee model.

The supersymmetric sine-Gordon theory is defined by the following action in euclidean space-time

$$
\begin{aligned}
S_{s s G}=\int_{-\infty}^{\infty} d y \int_{-\infty}^{\infty} d x & \left\{\frac{1}{2}\left(\partial_{x} \phi\right)^{2}+\frac{1}{2}\left(\partial_{y} \phi\right)^{2}-\bar{\psi}\left(\partial_{x}-i \partial_{y}\right) \bar{\psi}+\psi\left(\partial_{x}+i \partial_{y}\right) \psi-\right. \\
& \left.-\frac{m^{2}}{\beta_{s s G}^{2}} \cos \left(\beta_{s s G} \phi\right)-2 m \bar{\psi} \psi \cos \left(\frac{\beta_{s s G} \phi}{2}\right)\right\}
\end{aligned}
$$

where $\phi$ is the bosonic field and $\psi$ and $\bar{\psi}$ are the components of a Majorana fermion. The spectrum of the full quantum theory contains (anti-)soliton multiplets and bound state multiplets $\left(b_{j}, f_{j}\right), j=1,2, \ldots<\lambda$, $\lambda=2 \pi\left(1-\left(\beta_{s s G}^{2} / 4 \pi\right)\right) / \beta_{s s G}^{2}$, of masses (3.6) with $\beta=1 /(2 \lambda)$.

In all these examples, the bulk S-matrices and boundary R-matrices are of the general form discussed in sections III and IV [3, [, [1]. The detailed form of the reflection matrices was worked out in our recent paper [1].

We already mentioned that the thermodynamic Bethe Ansatz (TBA) is a very effective way to test the validity of conjectured S-matrices. While this analysis is more or less routine for diagonal S-matrices, the analysis for nondiagonal S-matrices is non-trivial and has to be studied on a case by case basis. Fortunately, $N=1$ supersymmetric integrable models can be mapped into the eight-vertex model at a special point, where they satisfy the so-called "free-fermion" condition, which allows to complete the TBA program. This was done in [5] for the super Yang-Lee case and in [6] for the more general perturbed superconformal field theories discussed in this section.

In the case of the supersymmetric sine-Gordon theory, we have been able to propose exact reflection matrices without knowing the boundary action. An interesting problem is then to find the boundary actions that correspond to these matrices. Inami, Odake and Zhang [7] have proposed two possible boundary actions that preserve integrability and supersymmetry. Their proposal is based on the study of the conserved charges, at the classical level, in the presence of a boundary. In [1] we established a connection between this proposal and our reflection matrices by looking at the weak coupling limit of the supersymmetric sine-Gordon model [1].

\section{TOPOLOGICAL CHARGES}

In the presence of topological charges, the anticommutator $\left\{Q_{+}, Q_{-}\right\}$changes to

$$
\left\{Q_{+}, Q_{-}\right\}=Z \text {, }
$$


with $Z \leq 2$. f We will adopt the following realization

$$
Q_{+}(\theta)=\sqrt{m} e^{\frac{\theta}{2}}\left(\begin{array}{ll}
0 & 1 \\
1 & 0
\end{array}\right), \quad Q_{-}(\theta)=\sqrt{m} e^{-\frac{\theta}{2}}\left(\begin{array}{cc}
0 & \mathrm{e}^{i \alpha} \\
\mathrm{e}^{-i \alpha} & 0
\end{array}\right), \quad Q_{L}(\theta)=\left(\begin{array}{cc}
1 & 0 \\
0 & -1
\end{array}\right),
$$

where $\cos (\alpha)=Z / 2$. Note that the case $Z=0$ is obtained when $\alpha=-\pi / 2$. Similarly to the case without topological charges we assume that the reflection matrices will be of the same factorized form as in (4.1).

Following the same approach as in the case without topological charges it is easy to see that the "supersymmetric" part of the reflection matrix will have the following form

$$
R_{B F}^{( \pm)}(\theta)=Z^{( \pm)}(\theta)\left(\begin{array}{cc}
\cosh \left(\frac{\theta}{2}+i\left(\frac{\pi}{4}+\frac{\alpha}{2}\right) \pm i \frac{\pi}{4}\right) & 0 \\
0 & \cosh \left(\frac{\theta}{2}+i\left(\frac{\pi}{4}+\frac{\alpha}{2}\right) \mp i \frac{\pi}{4}\right)
\end{array}\right) .
$$

At $Z=0$ this reduces to the reflection matrix in (4.5). Again we recall that this is the reflection matrix obtained by imposing that the boundary action preserves supersymmetry and integrability. Notice that we again have a universal ratio

$$
\frac{R_{b}^{ \pm}}{R_{f}^{ \pm}}=\frac{\cosh \left(\frac{\theta}{2}+i\left(\frac{\pi}{4}+\frac{\alpha}{2}\right) \pm i \frac{\pi}{4}\right)}{\cosh \left(\frac{\theta}{2}+i\left(\frac{\pi}{4}+\frac{\alpha}{2}\right) \mp i \frac{\pi}{4}\right)}
$$

Recently, Hollowood and Mavrikis [8] have proposed exact $N=1$ supersymmetric S-matrices for theories with non-zero topological charges. We expect that the reflection matrices (6.3) can consistently be combined with these new $S$-matrices, in the sense that together they form a solution of the BYBE.

\section{ACKNOWLEDGEMENTS}

We would like to thank Roland Köberle and Andreas Fring for useful discussions. One of us (MM) would like to thank the University of Amsterdam, where part of this work was done, for the warm hospitality. The research of KS was supported in part by the foundation FOM of the Netherlands.

[1] M. Moriconi and K. Schoutens, Reflection Matrices for Integrable N = 1 Supersymmetric Theories, Nucl. Phys. B487 (1997) 756-778, hep-th/9605219.

[2] L. Castillejo, R.H. Dalitz and F.J. Dyson, Low's Scattering Equation for the Charged and Neutral Scalar Theories, Phys. Rev. 101 (1956) 453-458.

[3] K. Schoutens, Supersymmetry and Factorizable Scattering, Nucl. Phys. B344 (1990) 665-695.

[4] C. Ahn, Complete S Matrices of Supersymmetric sine-Gordon Theory and Perturbed Superconformal Model, Nucl. Phys. B354 (1991) 57-84.

[5] C. Ahn, Thermodynamics and Form Factors of Supersymmetric Integrable Field Theories, Nucl. Phys. B422 (1994) 449-475, hep-th/9306146.

[6] M. Moriconi and K. Schoutens, Thermodynamic Bethe Ansatz for $N=1$ Supersymmetric Theories, Nucl. Phys. B464 (1996) 472-491, hep-th/9511008.

[7] T. Inami, S. Odake and Y.-Z. Zhang, Supersymmetric Extension of the sine-Gordon Theory with Integrable Boundary, Phys. Lett. B359 (1995) 118-124, hep-th/9506157.

[8] T. Hollowood and E. Mavrikis, The $N=1$ Supersymmetric Bootstrap and Lie Algebras, Nucl. Phys. B484 (1997) 631-652, hep-th/9606116.

\footnotetext{
${ }^{4}$ This $Z$ should not be confused with the prefactors $Z^{( \pm)}$.
} 